\title{
Pupil diameter in migraine and tension headache
}

\author{
PETER D DRUMMOND \\ From the Department of Neurology, Prince Henry Hospital, and the School of Medicine, University of \\ New South Wales, Australia
}

SUMmary Pupil diameter was measured in darkness and in dull and bright illumination in 39 migrainous patients and in 15 tension headache sufferers during headache. In 21 migrainous patients, measurements were repeated during the headache-free interval. Mean pupil diameter was smaller in patients with common migraine at the time of examination than in 20 nonheadache control subjects, and smaller on the symptomatic side in migrainous patients with unilateral headache. During the headache-free interval mean pupil diameter did not differ from values in nonheadache controls. These findings suggest that migraine is associated with a sympathetic pupillary deficit which is greater on the habitually-affected side.

Pharmacological studies of the pupil in migrainous patients have demonstrated a reduced response to agents causing release of neuronal stores of noradrenaline, and an exaggerated response to agents directly mimicking the action of noradrenaline. ${ }^{12}$ These findings indicate that migraine is associated with a pupillary sympathetic deficit and denervation hypersensitivity. This may be secondary to carotid artery dilatation and subsequent compression of the sympathetic plexus surrounding the artery, or could mirror impairment in central aminergic neuronal systems. $^{1}$

Pharmacological investigation of the laterality of pupillary deficits in a small number of patients with unilateral migraine has not demonstrated greater abnormality on the symptomatic side. Depletion of neuronal stores of noradrenaline by guanethidine in six patients with recurrent unilateral migraine resulted in similar pupillary miosis on the symptomatic and nonsymptomatic sides. ${ }^{1}$ Eighteen of 20 patients with migraine or cluster headache ${ }^{3}$ demonstrated anisocoria after cocaine eyedrops, but laterality of miosis was unrelated to side of headache in nine patients who were subject to unilateral headache. Gotoh et $\mathrm{al}^{4}$ reported that the pupils dilated equally on the symptomatic and nonsymptomatic sides in patients with recurrent unilateral migraine after instillation of

Address for reprint requests: Dr P D Drummond, Department of Neurology, The Prince Henry Hospital, Little Bay, 2036, New South Wales, Australia.

Received 24 September 1985 and in final revised form 30 April 1986. Accepted 19 June 1986
$1.25 \%$ adrenaline solution, but did not state how many patients were tested. Criteria for unilateral headache were not provided in any of these studies.

If a sympathetic deficit does form part of the pathophysiology of migraine, pupillary abnormalities could be exaggerated during headache. To determine whether transient or permanent changes in pupil diameter were associated with migraine, pupil diameter at various levels of illumination was examined during and between episodes of migraine, and compared with diameter in tension headache sufferers and nonheadache controls. In patients with unilateral headache, pupil diameter was compared on the headache and headache-free sides.

\section{Methods}

\section{Subjects}

Pupil diameter was measured in 54 headache patients and 20 control subjects of similar age and sex distribution (table). Diagnoses of migraine or tension headache were made on the basis of a standard clinical interview, supplemented by a neurological and general physical examination and, when indicated, laboratory tests to ensure that headache was not caused by a space-occupying lesion or other structural cause. Patients with serious visual impairment or a history of eye disease were not included in the study.

Migraine headache was preceded by focal neurological symptoms, or was associated with vomiting or with at least two other symptoms of migraine (nausea, dizziness, faintness, blurred vision or paraesthesiae during headache). Twenty two migrainous patients reported daily low-grade pain between attacks of migraine. In 19 patients with a clinical history of migraine, headache at the time of examination was not associated with the migrainous features listed above 
Table Sex distribution, laterality of headache and mean age in headache and control groups

\begin{tabular}{lcccccc}
\hline & & & \multicolumn{3}{c}{ Headache } \\
\cline { 5 - 7 } Group & $N$ & Male & Female & Unilateral & Bilateral & Age (yr) \\
\hline Tension headache & 15 & 6 & 9 & 0 & 15 & $47 \cdot 8$ \\
Migraine (nonspecific attack) & 19 & 6 & 13 & 17 & $41 \cdot 8$ & $44 \cdot 7$ \\
Migraine (migrainous attack) & 20 & 3 & 17 & 18 & 45 \\
Total & 54 & 15 & 39 & 35 & 19 & $39 \cdot 7$ \\
Nonheadache controls & 20 & 3 & 17 & & & \\
\hline
\end{tabular}

(termed "nonspecific" headache in the table to distinguish these patients from those with a clinical history of tension headache). Tension headache sufferers described a history of, and presented with, bilateral headache which was associated with few or no features of migraine; the headache recurred daily in 11 patients and was episodic in the other four patients. Twenty six of the 54 patients took prophylactic medication regularly, and 22 had taken some form of medication on the day of examination: analgesics (8), ergotamine tartrate (2), anti-emetic agents (5) and prophylactic medication (14).

Measurements were repeated during the headache-free interval in 21 migrainous patients, 11 of whom had been examined previously during migraine; the other 10 patients had been seen initially with nonspecific headache.

Twenty hospital staff of similar age and sex distribution to headache patients, but who reported 12 or fewer mild headaches per year and two or fewer severe headaches per year, served as nonheadache controls. A second pupillographic examination was carried out in 15 control subjects several days or weeks after the first measurement.

\section{Procedure}

Details of medication consumption, headache site and associated symptoms at the time of examination were recorded on a standard form. The patient then positioned his or her head on an adjustable chinrest and a fixed forehead rest in a small light-tight photographic chamber. An infrared flash

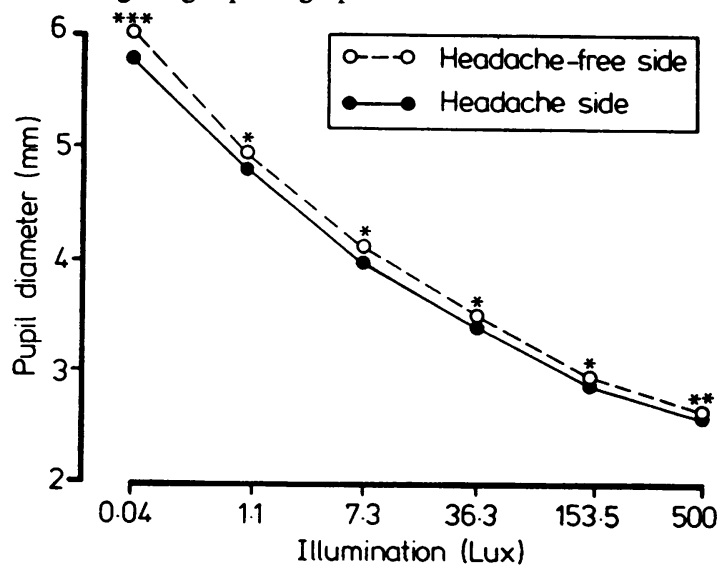

Fig Pupillary asymmetry in migrainous patients with unilateral common migraine or nonspecific unilateral headache. Pupil diameter smaller on the side of headache than on the headache-free side: ${ }^{*} p<0.05 ;{ }^{* *} p<0.01$; ${ }^{* * *} p<0.001$. recorded photographs of the patient's eyes on infrared film via a camera of fixed focal length housed behind a two-way mirror in the photographic chamber. Light from an evenly illuminated opaque white screen above the patient's head was reflected $90^{\circ}$ by the two-way mirror to shine into the patient's eyes. The patient was instructed to look at a black dot $56 \mathrm{~cm}$ away attached to the opaque screen. After 30 seconds of darkness, the screen was illuminated for $30 \mathrm{sec}-$ onds, the patient was told to stop blinking and a photograph was taken. After two practices, photographs were taken at each of six levels of illumination: 0.04 lux (darkness), 1·1, 7.3, $36 \cdot 3,153 \cdot 5$ and 500 lux, measured with a Manolux electronic Luxmeter at the position of the eyes in the photographic chamber. The six levels of illumination were presented randomly within each series of photographs. To average out random fluctuations in pupil diameter, three series of six photographs were taken for each patient.

Horizontal pupil diameter was later measured with calipers from the film negative magnified 8.0 times. Calibration was checked routinely by photographing a metal mm-graded ruler at the position of the patient's eyes in the photographic chamber.

Analyses of variance were used to test differences in average pupil diameter between patients with common migraine, tension headache and controls, to determine whether medication influenced pupil diameter, to assess changes in pupil diameter during and between episodes of common migraine, and to compare pupil diameter on the headache and headache-free sides in patients with unilateral headache. Because only two patients were examined during classical migraine their data were not included in statistical analyses and are reported separately.

\section{Results}

\section{Mean pupil diameter in relation to diagnosis and headache state}

Pupil diameter did not differ between nonheadache control subjects and migrainous patients during the headache-free interval, but was smaller in 18 patients with common migraine at the time of examination than in controls at every level of illumination (mean difference $0.4 \mathrm{~mm}, \mathrm{~F}(1,36)=4.37, \mathrm{p}<0.05)$. Pupil diameter did not differ between controls, tension headache sufferers or migrainous patients with nonspecific headache at the time of examination, or between medicated and unmedicated patients.

Mean pupil diameter decreased $0.1 \mathrm{~mm}$ in 15 control subjects who were retested after several days 
or weeks $(F(1,14)=5.72, p<0.05)$, and $0.2 \mathrm{~mm}$ in 10 migrainous patients who were retested several days or weeks after an episode of nonspecific headache $(F(1,9)=4 \cdot 29, p<0 \cdot 10)$; in contrast, no change in pupil diameter was observed in nine patients who were retested after an episode of common migraine. Pupil diameter in darkness was unrelated to headache state in one patient studied initially during classical migraine and was smaller by $0.4 \mathrm{~mm}$ during headache in the other patient with classical migraine.

\section{Pupillary asymmetry in unilateral headache}

At every level of illumination, the pupil on the side of the headache was significantly smaller than on the headache-free side $(F(1,31)=9.77, p<0.01)$ (fig), both for patients examined during common migraine $(p<0.05)$ and nonspecific headache $(p<0.05)$. In two patients with classical migraine, pupil diameter in darkness was smaller on the side of headache by $0.2 \mathrm{~mm}$ and $0.3 \mathrm{~mm}$ respectively, and this asymmetry persisted at low levels of illumination. Pupillary asymmetry was greatest in darkness and decreased progressively with increasing illumination (interaction between degree of asymmetry and illumination, $F(5$, $155)=5.86, \mathrm{p}<0.001$ )) (fig). Although 21 of 35 patients took prophylactic medication regularly, and 17 had taken one or more forms of medication on the day of examination, this did not influence pupil asymmetry.

At least $70 \%$ of headaches recurred habitually on the same side of the head in 32 of 35 patients; headache was on the customary side at the time of examination in all but one case. In this patient, the pupil was smaller on the habitually-affected side. In the three patients whose headaches recurred equally on either side, pupil diameter was symmetrical in one and smaller on the side of headache in two.

Miosis on the habitually-affected side was maintained in 17 migrainous patients who were retested during the headache-free interval.

\section{Discussion}

Pupillography demonstrated a subtle constriction of pupil diameter during common migraine. This constriction may be a physiological manifestation of the pupillary sympathetic deficit in migrainous patients demonstrated pharmacologically by Fanciullacci. ${ }^{1}$ Mean pupil diameter decreased on retesting in control subjects and in migrainous patients examined initially during headache associated with few or no migrainous symptoms, presumably because novel aspects of the experimental procedure caused greater autonomic activation during the first test. A reduction in pupil diameter during the headache-free interval was not observed in patients examined initially during common migraine, thus supporting the view that sympathetic pupillary activity was impaired. This deficit could be secondary to damage of sympathetic fibres in the head or neck, or could reflect an abnormality in regulation of central monoamine pathways. Pupil diameter did not differ from control values in tension headache sufferers or in migrainous patients who were examined during an episode of nonspecific headache or when headache-free.

In unilateral common migraine the pupil on the symptomatic side was smaller than on the headachefree side. This asymmetry was also observed in the absence of migrainous features (nonspecific headache), and persisted during the headache-free interval in migrainous patients whose headache recurred habitually on one side. Pharmacological investigations of patients with recurrent unilateral migraine have not demonstrated a unilateral sympathetic deficit on the symptomatic side, ${ }^{13}$ possibly because of the small number of patients studied (six and nine patients respectively). These studies should be extended to determine whether miosis on the side habitually affected by headache indicates a sympathetic deficit or "simple central" anisocoria. Greater pupillary asymmetry in darkness than in bright light makes the former possibility more likely.

The author thanks Professor J W Lance for his constructive suggestions during the developmental stages of this investigation and during the preparation of the manuscript, and Mr J W Duckworth for his assistance in assembling the photographic apparatus. This study was supported by the National Health and Medical Research Council of Australia and the J A Perini and Basser Trusts.

\section{References}

1 Fanciullacci M. Iris adrenergic impairment in idiopathic headache. Headache 1979;19:8-13.

2 Del Bene E, Poggioni M, Fanciullacci M. Iris adrenergic impairment in migraineous and hyperactive children. Int J Clin Pharmacol Res 1981;1:119-25.

3 Herman P. The pupil and headaches. Headache 1983;23:102-5.

4 Gotoh F, Komatsumoto S, Araki N, Gomi S. Noradrenergic nervous activity in migraine. Arch Neurol 1984;41:951-5.

5 Loewenfeld IE. "Simple central" anisocoria: a common condition, seldom recognised. Trans Am Acad Ophthalmol Otolaryngol 1977;83:832-9. 